\title{
Práctica docente en el aula multigrado rural de una población mexicana ${ }^{1}$
}

\author{
José Ángel Vera Noriega \\ Rosario Leticia Domínguez Guedea \\ Universidad de Sonora
}

\author{
Correspondência: \\ José Ángel Vera Noriega \\ Centro de Invest. en Alimentación \\ y Desarrollo \\ Carretera a la Victoria Km 0.6 - \\ Hermosillo Sonora \\ Apdo. Postal 1735 - México \\ e-mail: avera@cascabel.ciad.mx
}

\section{Resumo}

Con el objeto de presentar el escenario del aula multigrado en la zona rural en pobreza extrema se emplea una estrategia de clasificación estadística con el procedimiento por conglomerados. La muestra poblacional fue de 206 docentes que se clasificaron en base a datos de observación y reporte verbal de estrategias didácticas, control de grupo, planeación, evaluación y apoyo al aprendizaje, identificándose 5 tipos en la práctica docente. Los grupos se contrastan con los resultados en competencias académicas básicas de sus alumnos. Se señala la importancia de la diversidad en las estrategias didácticas y de manejo grupal, así como la variedad de elementos que los docentes emplean en su planificación y evaluación como la característica que permite identificar la diferencia entre los resultados de los alumnos. En la perspectiva de la política educativa vigente se analiza el impacto de los mecanismos de incentivos y capacitación docente como elementos para promover habilidades cognitivas sobre el proceso enseñanza-aprendizaje. Se resaltan las condiciones de la enseñanza multigrado en zona rural como elemento primordial para mediar la regulación de estrategias de control disciplinario y planeación de la práctica docente. Se crítica los sistemas actuales de compensación por la preponderancia de una visión administrativa de la enseñanza. Finalmente se enfatiza la utilidad de la clasificación de la práctica docente para posteriores acciones de capacitación específica a las condiciones de la zona rural, así como a distintos niveles de pericia de los profesores.

\section{Palavras-chave}

Tipología - Práctica docente - Zona rural - Enseñanza multigrado. ${ }^{1}$ Los autores agradecen al Sistema
de Investigación del Mar de Cortés
por el financiamiento otorgado para
la realización de este estudio. 


\section{Teaching practice in the multigrade rural classes in a Mexican village*}

José Ángel Vera Noriega

Rosario Leticia Domínguez Guedea

Universidad de Sonora

\author{
Contact: \\ José Ángel Vera Noriega \\ Centro de Invest. en Alimentación \\ y Desarrollo \\ Carretera a la Victoria $\mathrm{Km} 0.6$ - \\ Hermosillo Sonora \\ Apdo. Postal 1735 - México \\ e-mail: avera@cascabel.ciad.mx
}

'The authors wish to thank the Sistema de Investigación del Mar de Cortés for the financial support to this study.

\begin{abstract}
With the purpose of presenting a view of the multigrade classes in a rural area of extreme poverty, a statistical classification strategy is employed here proceeding by clusters. The sample population was made of 206 teachers, which were classified on the basis of data observed and verbal reporting of didactic strategies, group control, planning, and evaluation and support to learning, allowing the identification of five types in the teaching practice. The groups were contrasted with their students' results in basic academic competencies. The article highlights the importance of diversity in the didactic and group supervision strategies, and also of the variety of elements teachers employ in their planning and evaluation, as the characteristics that allow identifying the difference in students' results. Under the perspective of the current educational policy the text analyzes the impact of the mechanisms of incentives and teacher education as elements for the promotion of cognitive abilities for the teaching-learning process. The text emphasizes the conditions of the multigrade teaching in rural areas as a fundamental element in mediating the regulation of discipline control strategies and planning of teaching practice. It criticizes the current systems of compensation for their excessively managerial perspective of teaching. Finally, it emphasizes the usefulness of the classification of teaching practice for future training actions specific to the conditions of the rural area, and to the distinct levels of expertise of the teachers.
\end{abstract}

\section{Keywords}

Typology - Teaching practice - Rural area - Multigrade teaching. 


\section{Introducción}

El aula multigrado, bastante frecuente en la zona rural, esta recobrando la importancia de antaño. Existen estudios que muestran algunas ventajas didácticas al trabajar con niños de diferentes edades y complejidad de competencias en donde un paradigma colaborativo con una prepuesta de apoyo a la diversidad y desarrollo de habilidades de tolerancia y entendimiento genera resultados académicos, éticos y estéticos en los niños.

La práctica docente en el aula multigrado implica además de condiciones y relaciones, procesos reales de trabajo que se construyen a partir de la negociación entre sujetos (profesores, alumnos, padres de familia) y "conocimientos efectivamente integrados a la práctica docente" (Rockwell, 1990, p.32-34), conocimientos que no están siendo adquiridos en el proceso formal sino mas bien en el transcurrir de los años de experiencia.

Algunas aproximaciones sobre la evaluación de la práctica docente en el aula tradicional homogenea unigrado, parten de habilidades de tipo instrumental, incluyendo en éstas destrezas particulares para la implementación de estrategias didácticas que se definen por su carácter manual y situacional (Galo de Lara, 1990 citado en Alvarez, 1997); algunos bajo la lógica de competencia en el ámbito del ejercicio profesional (Zarzar, 1994, Gallart; Jacinto, 1995) consideran las habilidades universales del docente en un marco de flexibilidad y adecuación a las necesidades particulares; y finalmente, algunos otros clasifican el desempeño en términos de competencias de interacción social (Gajardo; De Andraca, 1992; Cazares, 1995) considerando aspectos de la personalidad del docente como elementos en la relación con sus alumnos y la comunidad educativa y en ese sentido, son esos los elementos a considerar en la valoración del docente (Velásquez, 1996).

Fernández (1994) señala el carácter operacional (instrumental) y secuencial que guardan entre sí las principales tareas de la práctica docente como son las actividades de programación, las competencias técnico pedagógicas de impartir las clases, explicar las lecciones y ayudar a los alumnos a aprenderse temas; y la tarea de examinar, asignar notas o evaluar a los alumnos.

Algunos estudios relevantes para comprender la diversidad y la diferenciación de las prácticas de enseñanza ha sido objeto de investigaciones que van desde el orden psicopedagógico (Ahumada, 1992; Fernández, 1994) hasta una perspectiva más sociológica de la práctica docente (Schmelkes; Muñoz, 1982; Rockwell, 1990; lbarrola; Silva; Catelán, 1997; Ezpeleta; De Weiss, 2000).

La evaluación de la práctica docente en términos de los parámetros normativos que delimita un currículo al interior de un sistema educativo particular es incompleta si no obtenemos datos de la forma en la cual los ejercicios propios de planeación, evaluación y didáctica promueven el niño las competencias básicas que se propone el sistema educativo nacional. Esto ha de suponer que las actividades que descuidan por los planes y programas de estudio tendrán como resultado una ejecución académica adecuada en un niño promedio. En este escenario ideal aún nos falta por conocer cómo se define un niño promedio y una ejecución adecuada, las habilidades docentes promovidas primero en la escuela normal y después en los programas de capacitación del magisterio que tratan de homogenizar las competencias del profesor al sistema educativo nacional. Las diferencias entre las prácticas de profesores estarán entonces más vinculadas al escenario escolar y al tipo y número de alumnos que a las competencias obtenidas en su proceso de formación o capacitación. Lo anterior no significa que se suponga un buen grado de especialización de habilidades y competencias docentes sino que debemos evaluarles en relación con la normatividad del programa, atentos a las diferencias que se encuentran en el aula, tanto físicas como de organización, que introducen 
elementos de dificultad accesoria que al comparar dos profesores con las mismas habilidades en dos contextos áulicos con distinto grado de organización (número de niños, número de grados, diversidad étnica y otros) generan resultados distintos.

En la evaluación de las habilidades docentes predominan tres enfoques: el contraste de habilidades y competencias dentro del grupo; la comparación entre grupos, ambos vinculados aun criterio o a una norma y finalmente el enfoque de resultados en el que las habilidades se miden con relación a criterios de ejecución de las competencias básicas académicas, más allá de criterios subjetivos de actitudes, opiniones, promedios escolares o puntuaciones colaterales de organismos de evaluación externa.

En este último enfoque no existen datos que nos permitan conocer el estado actual de la práctica docente en la zona rural, fundamentalmente en la escuela multigrado. Este análisis resulta importante debido a que será posible ensayar una metodología cuantitativa para clasificar a los profesores en términos de su comportamiento docente o sea en términos de la diferencia entre ellos, esto es elaborar conjuntos nominales y después compararlos en términos de los resultados obtenidos en la ejecución académica del niño.

La construcción de tipología o la caracterización de la práctica docente fusiona las ventajas que ofrecen los modelos de evaluación tradicional y de los estudios sociológicos centrados en el docente (Mckinney, 1968). La construcción de tipologías tiene una finalidad programática en el campo de la capacitación y mejoramiento del magisterio, así como los ajustes de las unidades en el aula, anticipando diferencias muy tenues entre los grupos, medir el papel que juegan los factores diferenciales del aula en los resultados obtenidos por los alumnos. Es un camino sistemático y pertinente para establecer un diagnóstico; la tipología, como método, es capaz de incluir las reglas de la sistematicidad y ofrecer un panorama objetivo y real del evento; así también con relación a los estudios centrados en la práctica docente, la tipología se convierte en una propuesta metodológica alternativa, una forma diferente de realizar el diagnóstico y la descripción de las condiciones y variables relacionadas a la práctica. (Hair Anderson; Tatham; Black, 2000).

Un primer planteamiento inductivo, señala la importancia de las variables de capacitación y formación y las condiciones físicas y organizacionales del aula; la segunda pregunta de trabajo se establece con relación a los aspectos que promuevan la diferencia entre el tipo de práctica docente de un profesor a otro y los resultados obtenidos por los alumnos; así mismo como describir de que forma se vinculan las condiciones físicas y organizacionales del aula en la diferencia obtenida en los resultados de los alumnos.

\section{Método}

Se aplicaron tres instrumentos para la valorar la práctica docente a una muestra de 206 profesores que trabajaban en la zona rural del sur del Estado de Sonora. La selección se realizó atendiendo a los criterios del estudio de marginación y pobreza del Centro de Investigación en Alimentación y Desarrollo A. C. (Camberos; Genesta; Huescas, 1994) y al número de ciclos escolares que atiende. Se consideraron establecimientos escolares tanto de adscripción federal como estatal, en cualquiera de los turnos matutino o vespertino.

La muestra de escuelas de la zona rural en pobreza extrema se obtuvo siguiendo las fórmulas para obtener una muestra representativa (Sierra Bravo, 1995) al 95\% de confiabilidad, $5 \%$ de error, $\mathrm{P}=.5$ y $\mathrm{q}=.5$. Los profesores fueron seleccionados de un listado de la población y evaluados en sus aulas de los grupos seleccionados.

El promedio de edad de los 206 docentes participantes fue de 39 años y la antigüedad en el magisterio una media de 16 años. El 36\% ( $n=74)$ de los participantes eran docentes de sexo femenino y el 64\% ( $n=132)$ 
de sexo masculino. El 40\% ( $\mathrm{n}=82)$ del total de docentes participantes reportó como último grado de estudio la normal básica en el ámbito técnico; el 16\% ( $n=33$ ) la normal a nivel licenciatura y el 44\% ( $n=91$ ) haber cursado alguna especialización en la Universidad Pedagógica Nacional.

El 83\% de los maestros ( $n=171)$ estaban adscritos a escuela multigrado, siendo el 16\% (n =27) los que atendían seis grados a la vez; 10\% $(\mathrm{n}=17)$ atendían cinco grados; 7\% $(n=12)$ a cargo de cuatro grados; 35\% ( $n=60)$ con tres grados; 30\% ( $n=51)$ atendiendo a dos grados. El $19 \%(n=39)$ restante eran docentes de zona rural sin embargo atendían un grado por grupo.

El 28\% de los profesores pertenecian ( = 58) a la comunidad de Álamos, el 25\% (n =51) al municipio de Navojoa; el 20\% ( $=42)$ en Huatabampo; el 19\% $(\mathrm{N}=40)$ en Etchojoa; quedando un $7 \%(n=15)$ en comunidades del municipio de Cajeme.

\section{Instrumentos}

\section{Guía de observación del desempeño de los docentes}

El instrumento consta de 16 reactivos de tipo ordinal que señalan condiciones referentes al manejo de contingencias y manejo didáctico del docente para la conducción y control disciplinario del grupo, incluye el registro de estrategias de evaluación utilizadas por el docente hacia el comportamiento de los alumnos en alguna actividad.

Los reactivos de este instrumento delimitan las observaciones en los siguientes términos: a) aseo y distribución de alumnos en el aula; b) recursos utilizados por docentes y alumnos; c) actividades realizadas durante la clase; d) manejo de contingencias; e) monitoreo y retroalimentación de conducta de alumnos; f) movilidad del docente; g) participación en clase (docente/alumnos); h) conducta del docente hacia los alumnos. Después de los primeros 15 minutos y habiendo terminado un período de ajuste del grupo de niños a la presencia del observador, se iniciaba la observación con una duración de 15 minutos. Al finalizar el registro, el observador hacía comentarios anecdóticos sobre sucesos que ocurrieran durante la observación.

\section{Entrevista sobre la práctica docente}

El instrumento consta de 35 reactivos de tipo ordinal, por medio de los cuales se recopila información referente a los siguientes aspectos:

Planeación: a) tiempo estimado para ejecución de planes; b) horas dedicadas para su preparación; c) formulación de objetivos; d) elementos considerado durante la planeación; e) recursos didácticos utilizados.

Práctica sobre evaluación: a) diferencia entre evaluación y calificación; b) estrategias utilizadas para evaluar; c) frecuencia con que evalúa; d) estrategias utilizadas para calificar; e) frecuencia con que califica; f) estrategias utilizadas ante resultados incorrectos de los alumnos.

\section{Participación de padres de familia}

Este instrumento está compuesto por 10 reactivos de tipo ordinal por medio de los cuales se busca recaudar información referente al uso de los recursos económicos del Apoyo a la Gestión Escolar (AGE), así como los criterios seguidos para otorgar incentivos a docentes y el apoyo por parte de los padres de familia en su labor docente y el aprendizaje de los niños.

\section{Estandarización del personal para levantamiento de datos}

Se formaron 4 equipos de investigadores con 3 miembros cada uno. Los 12 participantes eran psicólogos con experiencia en trabajo de campo.

Las formas de control y confiabilidad de las observaciones y aplicación de los instrumentos se realizaron con todos los investigadores 
participantes; para ello tuvieron efecto los siguientes momentos del entrenamiento: a) El conocimiento del instrumento: implicaba la lectura y aclaración de dudas sobre el significado de términos y estructura de los reactivos; b) El modelamiento de aplicación: implicaba la representación de una aplicación, por parte de un investigador experto, acentuando momentos críticos de la entrevista, guía o examen del niño, explicando el procedimiento adecuado en caso de que algún evento imprevisto se presentaran en campo; c) Aplicación con un grupo piloto: a fin de ensayar el repertorio verbal y conductual al momento de dirigir la aplicación del instrumento, se recurrió a localizar comunidades con características similares a la población blanco, pero en condiciones ingenuas al instrumento. La aplicación se hacía por parejas de investigadores; d) Ensayo del procedimiento de confiabilidad: Posterior a la aplicación del instrumento, en el grupo piloto cada investigador contrastó con su par las observaciones y anotaciones realizadas, a fin de obtener el índice de confiabilidad a partir de los acuerdos en las observaciones o selección del tipo de respuestas en la entrevista; e) Procedimiento de confiabilidad en campo: Cada investigador realizó al menos un ensayo de aplicación de cada instrumento con un par observador, a fin de realizar la prueba de confiabilidad con la población real.

Cada investigador se especializó en la aplicación de un instrumento, pese a que tuviera el conocimiento y la habilidad para aplicar todos los instrumentos; sin embargo se tomó esa medida para optimizar tiempo en campo.

\section{Procedimiento para la guía de observación de la práctica docente}

Para aplicar la guía de observación se procedió de la siguiente forma: a) se designaron los primeros 15 minutos después de ingresar al aula para un tiempo de ajuste a las condiciones del aula y habituación de los alumnos hacia las personas extrañas; b) posteriormente se iniciaba el periodo de observación, con una duración de 25 minutos, identificando cada una de las categorias que el instrumento plantea. En caso de que los procesos implicaran una secuencia más prolongada que el tiempo de observación, se anotaba las etapas que se observaron y se prolongaba el tiempo de observación mientras durara el proceso c) el registro de las categorías observadas se realizaba en el momento en que iniciaba la interacción; d) al final del tiempo de observación, el investigador realizaba un anecdotario describiendo las condiciones generales del ambiente en el aula, puntos relevantes en la interacción afectiva-social del maestro con los alumnos y viceversa.

\section{Procedimiento para la aplicación de la medida de evaluación a los alumnos}

Después de haber seleccionado a los alumnos con que se trabajaría así como haber señalado el lugar de trabajo, las actividades seguían la secuencia que a continuación describe el procedimiento de la evaluación a alumnos: se armaba la porta rota folio con las hojas que contenían la ampliación de los mismos ejercicios que los niños realizarian en los cuadernillos de trabajo, con el fin de controlar el ritmo del grupo y optimizar el tiempo disponible. Después de escuchar 2 veces el cuento se inicia la lectura de las instrucciones y se solicita a los alumnos que respondan los ejercicios relativos a ello. Cuando terminan, se pasa a la siguiente instrucción y así sucesivamente.

\section{Dimensiones de la práctica docente}

Con el objeto de integrar los datos obtenidos por el reporte verbal y la observación se consideraron las siguientes categorias analítico-conceptuales.

a) Estrategias didácticas: escala intervalar que señala la variedad y cantidad los recursos materiales y didácticos que el docente emplea para el desarrollo de la clase. Se obtiene a partir de la información que genera la guía de observación de la práctica docente.

b) Estrategias de manejo de grupo: escala 
intervalar que señala la variedad y cantidad de las estrategias de detección y corrección del comportamiento inadecuado, así como la conducción del grupo en el aula. Se complementa con el análisis de contenido de los registros anecdótico realizados durante la clase, en la guía de observación de la práctica docente. c) Habilidades de planeación: escala intervalar que señala la variedad y cantidad de los tiempos dedicados a la preparación de cada uno de los elementos de planeación, la variedad de estos elementos y la estimación que tiene el docente para la implementación de sus planes. Se obtiene a partir de la infor-mación que genera la entrevista sobre la práctica docente.

d) Habilidades de evaluación: escala intervalar que señala la variedad y periodicidad de las estrategias que el docente utiliza para evaluar el desempeño y logro de sus alumnos

e) Habilidades de apoyo para el aprendizaje: escala intervalar que indica la variedad y la cantidad estrategias que el docente emplea para vincularse con los padres de familia y solicitar apoyo para el aprendizaje de los alumnos.

\section{Resultados}

\section{Procedimiento para la obtención de conglomerados}

La conformación de conglomerados se realizó siguiendo el procedimiento de un análisis clúster (Hair et al., 2000), para elegir los conglomerados según la claridad de la descripción del cluster y su aplicación práctica, los datos fueron sometidos a un procedimiento QUICK CLUSTER
(SPSS, 2002). El primer paso de este método implica la conversión de los valores de media obtenidos por los sujetos en cada uno de las dimensiones de la práctica docente en valores $\mathrm{Z}$; el segundo momento es la selección del método para formar conglomerados así como la toma de decisión acerca del número de soluciones cluster que por teoría o evidencia empírica se supone para esta población. En este caso se realiza el procedimiento $\mathrm{K}$ - means para la formación de conglomerados, de los que se desprende información acerca de: a) el número de casos por conglomerado; b) la distancia de cada caso al centro del conglomerado, c) la distancia de los centros de conglomerados finales $y, d$ ) la significación estadística de las diferencias de conglomerados.

La tabla 1 muestra los valores de media estandarizadas, obtenidos por los sujetos pertenecientes a los distintos conglomerados con relación a las dimensiones de la práctica docente. En la medida que la puntuación obtenida se aleja de cero, el valor indica que existe mayor particularidad del conglomerado para la práctica de esa dimensión; el valor negativo señala que el valor obtenido es por debajo de la media esperada para esa dimensión.

Los datos apoyan la posibilidad de distinguir la práctica docente de la zona rural del sur del estado en 5 distintos grupos; 2 de ellos notablemente delimitados, con puntuaciones extremas; uno con puntuaciones regulares y dos grupos que guardan características particulares pero también comparten características con el grupo regular. Lo que sugiere distintas necesidades y condiciones laborales.

Tabla 1. Valores de media estandarizada en las dimensiones de la práctica docente

\begin{tabular}{|l|r|r|r|r|r|}
\hline Dimensión / cluster & \multicolumn{1}{|c|}{1} & \multicolumn{1}{c|}{$\mathbf{1}$} & \multicolumn{1}{c|}{$\mathbf{3}$} & \multicolumn{1}{c|}{4} & \multicolumn{1}{c|}{5} \\
\hline Habilidades de planeación & .43 & -.02 & -.36 & -2.45 & .54 \\
\hline Habilidades de evaluación & .47 & -.00 & .17 & -2.88 & .18 \\
\hline Estrategias didácticas & 1.51 & -.82 & .52 & -.05 & .07 \\
\hline Estrategias de manejo grupal & 1.57 & -.88 & .73 & .08 & -.05 \\
\hline Apoyo al aprendizaje & 1.33 & -.32 & -.76 & -1.05 & .62 \\
\hline Valor F & 37.88 & 40.67 & 60.59 & 97.54 & 53.95 \\
\hline significancía & .000 & .000 & .000 & .000 & .000 \\
\hline
\end{tabular}


Tabla 2. Validez predicativa de los conglomerados a partir de variables adicionales

\begin{tabular}{|l|c|c|c|c|c|c|c|}
\hline Variables adicionales / cluster & $\mathbf{1}$ & $\mathbf{2}$ & $\mathbf{3}$ & $\mathbf{4}$ & $\mathbf{5}$ & $\mathbf{f}$ & $\mathrm{P}$ \\
\hline Media de español & 35.65 & 23.59 & 53.88 & 61.16 & 78.37 & 256.154 & .000 \\
\hline Media de matemáticas & 41.44 & 26.08 & 57.12 & 33.93 & 53.67 & 92.120 & .000 \\
\hline
\end{tabular}

La tabla 2 muestra los datos obtenidos para evaluar la relación con los resultados de los alumnos en competencias académicas básicas para los conglomerados. En este caso son las variables producto (media de puntaje alcanzado por los alumnos en habilidades de español y matemáticas).

La solución de 5 conglomerados muestra diferencias estadísticamente significativas sobre estas dos variables de resultado. Los resultados muestran al conglomerado 5 que obtuvo valores aprobatorios (mayor a 60) en habilidades de español, a su vez, es el conglomerado que obtuvo valores positivos en la mayoría de las dimensiones de la práctica docente y con puntuaciones equilibradas en todas las dimensiones.

La tabla 3 ofrece un perfil descriptivo de los conglomerados con relación a las variables de capacitación y formación de los profesores. Se han incluido sólo aquellas variables que resultaron con un nivel de significación del .001, a partir de un procedimiento de CROSSTABS (SPSS, 2002) para tabular los valores de los conglomerados contra algunas variables categóricas, en este caso, algunas variables de identificación (variables insumo) que permitieran asegurar la correspondencia de las agrupaciones-cluster dentro de las expectativas teóricas y empíricas. Se ha seleccionado un nivel alto de significancía por la necesidad de acentuar la pertinencia de estas variables como parte de la definición de la tipología de docentes.

Con base a los datos de las tres tablas anteriores, es posible definir la tipología de la práctica docente en la zona rural del sur del Estado. Esta tipología destaca cinco dimensiones de la práctica docente basada en la diversidad de estrategias (es decir la cantidad y combinación de elementos incorporados en cada quehacer del docente), generando un continuo intervalar con un cero convencional. Los incrementos en los valores representan diferencias en estilos para cada categoría de la práctica didáctica y son

Tabla 3. Construcción de perfiles a partir de variables adicionales

\begin{tabular}{|c|c|c|c|c|c|c|c|}
\hline Variables adicionales /cluster & 1 & 2 & 3 & 4 & 5 & $\begin{array}{l}\text { Chi cuadrado } \\
\text { de Pearson }\end{array}$ & $P$ \\
\hline $\begin{array}{l}\text { Último grado de estudios } \\
\text { Normal Básica /Lic. Ajena } \\
\text { Normal Superior Lic. } \\
\text { UPN Completa o Lic. en Educación. }\end{array}$ & $\begin{array}{l}6 \\
3 \\
13\end{array}$ & $\begin{array}{l}38 \\
6 \\
28\end{array}$ & $\begin{array}{l}13 \\
5 \\
25\end{array}$ & $\begin{array}{l}2 \\
7 \\
1\end{array}$ & $\begin{array}{l}23 \\
12 \\
24\end{array}$ & 34.294 & .000 \\
\hline $\begin{array}{l}\text { Tipo de organización de la escuela } \\
\text { completa } \\
\text { multigrado }\end{array}$ & $\begin{array}{l}4 \\
18\end{array}$ & $\begin{array}{l}11 \\
61\end{array}$ & $\begin{array}{l}11 \\
32\end{array}$ & $\begin{array}{l}6 \\
4\end{array}$ & $\begin{array}{l}3 \\
56\end{array}$ & 21.468 & .000 \\
\hline $\begin{array}{l}\text { Número de grados que atiende } \\
\text { atiende un grado } \\
\text { dos grados } \\
\text { tres grados } \\
\text { cuatro grados } \\
\text { cinco grados } \\
\text { seis grados }\end{array}$ & $\begin{array}{l}5 \\
7 \\
7 \\
0 \\
0 \\
3 \\
\end{array}$ & $\begin{array}{l}11 \\
12 \\
22 \\
6 \\
13 \\
8\end{array}$ & $\begin{array}{l}12 \\
12 \\
11 \\
2 \\
0 \\
6\end{array}$ & $\begin{array}{l}8 \\
2 \\
0 \\
0 \\
0 \\
0 \\
\end{array}$ & $\begin{array}{l}3 \\
18 \\
20 \\
4 \\
4 \\
10 \\
\end{array}$ & 55.029 & .000 \\
\hline $\begin{array}{l}\text { Disponibilidad de apoyo a la gestión escolar } \\
\text { no } \\
\text { si }\end{array}$ & $\begin{array}{l}14 \\
8\end{array}$ & $\begin{array}{l}30 \\
42\end{array}$ & $\begin{array}{l}26 \\
17 \\
\end{array}$ & $\begin{array}{l}10 \\
0\end{array}$ & $\begin{array}{l}18 \\
41\end{array}$ & 24.057 & .000 \\
\hline
\end{tabular}


clasificados en categorias que van de mayor a menor diversidad utilizando como criterio los cuartiles obtenidos de la sumatoria de los puntajes del número de estrategias o modos utilizados en la actividad docente.

\section{Tipologia \\ El tipo 1}

El tipo 1 de práctica docente que ejercitan los 22 maestros, integra mayores elementos en la implementación de estrategias didácticas, en las de manejo de grupo, en las habilidades de planeación y en las de evaluación, en este sentido demuestra, en conjunto una práctica más diferente (en términos de su cercanía a los valores de media y centro del conglomerado) con relación a la práctica que desempeñan los profesores de los otros 4 conglomerados. Se puede agregar a sus características que la mayoría está compuesto por docentes con nivel licenciatura, adscritos a una escuela multigrada, quienes atienden entre dos y tres grados por grupo y presentan valores bajos de media en estrategias de vinculación relacionadas con la gestión escolar.

Su práctica se caracteriza por desarrollar el mayor número de estrategias para cualquiera de las dimensiones de la práctica docente; es decir, desarrollan una práctica más variada y diferente a las rutinas en comparación del resto de la población docente; pese a lo que pareciera una práctica más adecuada y que promueve mayor cantidad de situaciones de estimulación, los alumnos de estos maestros no obtienen valores de media aprobatoria en las asignaturas de matemáticas y español.

\section{El tipo 2}

El tipo 2 de práctica docente que agrupa a 72 maestros es considerablemente heterogéneo en cuanto a la preparación académica de los docentes que lo constituyen y agrupa la mayor cantidad de docentes con preparación en la Universidad Pedagógica Na- cional (UPN), aunque también es el grupo con más docentes que tienen la normal básica. En este grupo está la gran mayoría de los maestros adscritos a la organización multigrado y concentra también a los docentes que imparten más de dos grados por grupo. Su práctica se caracteriza por desarrollar el menor número de estrategias para cualquiera de las dimensiones de la práctica docente; es decir, desarrollan una práctica restringida en variedad y cantidad de recursos. Los alumnos de estos maestros obtienen los valores de media más bajos en las asignaturas de matemáticas y español, que el resto de la población observada.

\section{El tipo 3}

El tipo 3 de práctica docente incluye los 43 maestros en su mayoría egresados de la UPN y adscritos a escuelas multigradas, atienden a pocos grados por grupo (uno a tres) y no disponen de apoyo para la gestión escolar. Se caracterizan por desarrollar una compensación entre las prácticas de planeación y evaluación con relación a las de manejo de grupo y estrategias didácticas.

\section{El tipo 4}

El tipo 4 de práctica docente incluye a los 10 maestros que atienden un grado por grupo, con estudios de especialización en la normal superior, y sin disponibilidad de apoyo a la gestión escolar. Su práctica se caracteriza por desarrollar una compensación entre las prácticas de planeación y evaluación y las prácticas de manejo de grupo y estrategias didácticas.

\section{El tipo 5}

El tipo 5 de práctica docente que incluye a los 59 maestros con formación de normal básica y UPN, lo que significa una heterogeneidad en las habilidades prácticas y teóricas. Reúne gran parte de los docentes 
adscritos a escuelas multigradas, donde atienden en su mayoría a 3 o 2 grados por grupo, contando con apoyo para la gestión escolar. Desarrollan distribuyen los tiempos y recursos para la planeación y evaluación así como de manejo de grupo y estrategias didácticas de forma equitativa, esto sus puntuaciones en estas dimensiones son con valor positivo y una magnitud similar.

\section{Discusión}

La edad y el sexo del profesor no promueven diferencias significativas para la tipología de la enseñanza, esto coincide con un estudio realizado (De la Torre, 1993) para la conformación de estilos docentes hacia la innovación. Esto contribuye a una primera conjetura: no es el variable sexo o edad lo que distingue la característica de la práctica docente.

Sin obviar la incidencia de los factores relacionados con las variables del alumno, que también han sido demostrados (González et al., 1998; Vera; Serrano, 1998; Vera et al., 1999; Bazán, 2001) para explicar los logros en las áreas de matemáticas y español, los datos y la predictibilidad de los conglomerados sugiere que el ejercicio equilibrado de la práctica docente en términos del tiempo invertido y variedad de estrategias implementadas, puede promover la posibilidad de éxito al menos en una de las asignaturas básicas de la educación primaria; De lo contrario una descompensación (es decir desarrollar unas habilidades más que otras) puede propiciar efectos negativos para el aprendizaje debido a que, mientras una planeación con gran variedad de elementos se puede tornar en un brumoso proceso de apego institucional, las habilidades no ejercitadas de manejo de grupo pueden ser la clave para retomar la conducción efectiva del grupo promoviendo espacios educativos ordenados que faciliten el contacto con los eventos académicos que irán formando los repertorios académicos básicos en el niño.

Algunas variables, específicamente la de apoyo a la gestión escolar y el nivel académico de los profesores, parecen agudizar las deficiencias en la práctica docente. Esto es, mientras que el conglomerado 1 y 5 obtienen valores positivos y muestran estrategias de vinculación con la comunidad, solo los profesores que del conglomerado 5 que cuentan con apoyo de AGE y reflejan puntuaciones positivas en una de las asignaturas evaluadas. Lo anterior sugiere que el equipamiento a las escuelas que contribuya a la organización de la infraestructura y provea de espacios más adecuados para el desarrollo cognitivo, no solo en el aula, si no en un ambiente enriquecido con mejores servicios, probabiliza un desempeño favorable en los estudiantes.

Este estudio acerca de la tipología de la práctica docente en la zona rural considera profesores que laboran en una diversidad de condiciones, una de ellas, quizá de las más importantes es que se desarrollan en grupos multigrados o unigrado, de ahí que resulta interesante la capacidad predictiva de los conglomerados, en tanto que la dinámica que se sugiere a partir de las condiciones que implica el Tipo 4, es por una parte compartida por el resto de los profesores rurales, pero por otra privativa de los profesores que están al frente de un solo grado.

Se considera que la tipología de la práctica docente ofrece una forma de aproximarse a la clasificación de la práctica docente, para posteriormente ser evaluado, analizado o intervenido considerando al menos los parámetros básicos que resultaron importantes: las estrategias didácticas, las de manejo de grupo, así como las habilidades de planeación y evaluación y que no conviene solo tomarlos como indicadores sino que obligan a la reflexión acerca de cuales son los procesos que generan su aparición o inhibición.

La tipología de la práctica docente permite bosquejar algunas líneas de intervención para la mejora de las acciones compensatorias en la zona rural. El tipo 5 de docentes es quien realiza una práctica equilibrada, no obtiene los mayores puntajes y mantiene una proporción 
entre lo que hace y lo que planea. Probablemente el tiempo que designa a la planeación es el suficiente para anticiparse a la demanda situacional. Probablemente con esos criterios también cumple en el sentido institucional de la planeación. Al contrario el tipo 1, que pareciera tener un desempeño adecuado, no ofrece resultados aprobatorios, lo que sugiere que el tiempo que dedica a la planeación es mayor al tiempo real de trabajo con los alumnos.

El ensamblaje de las categorías y las variables que componen al tipo no deben perder la lógica ni la propuesta teórica, esto es, el tipo 4 también produce calificaciones aprobatorias, sin embargo, su práctica se caracteriza por mayor control situacional que estrategias analíticas, en este caso, el profesor está mas a la disponibilidad en que ocurran los sucesos y no se provee de un sistema de retroalimentación que le permita orientar su práctica ni la de sus alumnos. Adicionalmente, la capacidad en estructurar rutinas y sistematicidad en la práctica docente permitirá replicar y analizar con mayor detenimiento los eventos que ocurran en el aula.

¿Es entonces el tipo 5 el profesor funcional para la zona rural? Dadas las condiciones de pobreza extrema y de aislamiento profesional, se considera que una práctica funcional debe ir más allá de la evidencia en los índices de calificación, seguramente existen mejores indicadores del aprendizaje que los utilizados en este estudio; sin embargo, los indicadores y procesos utilizados para estudiar la práctica docente, en su coherencia metodológica y práctica sugiere que bajo esas condiciones es más funcional mantener una práctica equilibrada en tiempo y esfuerzo.

Esta última consideración es importante en términos de los criterios de evaluación a los docentes, lo que conlleva a reflexionar sobre las condiciones laborales y estructurales en que los profesores se ven inmersos cotidianamente y la práctica institucional de evaluación que privilegia indicadores de administración de tiempo a la planeación llenado de formulario y planes de seguimiento.
En ese sentido, la capacitación que se ofrece a los docentes de esa zona, tal como fue diseñada, parece requerir una reconsideración de las demandas regionales, asi como partir del conocimiento no sólo de las prácticas de enseñanza del docente, si no de sus prácticas de aprendizaje, adopción de esquemas y conceptuación de su función docente (Fernández, 1994) a fin de promover tipología de docentes funcionalmente apegados a la planeación en escuelas multigrados que permitan construir ambientes curriculares y contextuales más organizados, evitando planeaciones burocratizadas y promoviendo planeaciones más analíticas y enriquecidas con elementos para la estimulación del aprendizaje de los alumnos.

Acorde a la idea anterior, los resultados coinciden con la tipología propuesta, ya que el tipo 1 que es el profesor más apegado a una planeación institucional no produce resultados aprobatorios, probablemente por el tiempo asignado y el tipo de control situacional que ejerce, donde posiblemente el resultado se ve en ese momento, pero no perdura. Pero por otro lado ¿Es entonces que los recursos de capacitación deberán orientarse a profesores de tipo 2 o 4 que no han asimilado el proceso de planeación para escuelas multigrados y dedican poco tiempo para ello lo que se traduce en situaciones en el aula poco estructuradas? La tipología ofrece la siguiente respuesta a manera de conjetura.

La planeación y evaluación no ocurren en forma aislada al resto de los quehaceres del docente, si el docente percibe mayor control y progreso del grupo ejerciendo de manera situacional formas de control y dinámica en el grupo, aunque no lo plantee en un documento, recurrirá más a soluciones pragmáticas que a la reflexión de la programación, realizando probablemente planeaciones y evaluaciones rutinarias que le permitan presentar resultados en la estadística institucional, pero que no necesariamente funjan como medios de retroalimentación.

Dos de estos factores comprenden un repertorio de comportamientos que pueden ser 
mejorados y conducidos para perfeccionar perfiles de acuerdo a las necesidades del contexto: la preparación profesional implica considerar los saberes del docente desde su formación inicial, aquellos que incorpora a su práctica cotidiana en materia de contenidos programáticos y metodológicos para desarrollar el proceso de enseñanza aprendizaje mediante materiales curriculares vigentes; mientras que desempeño profesional, se considera como las acciones que realiza el profesor para organizar y conducir el proceso de enseñanza aprendizaje, abarcando también las formas de organización y relación con la comunidad escolar y en general, que promuevan efectos en el aprendizaje, en ese sentido es conveniente considerarlos como factores de evaluación ya que significan dos importantes condiciones de la situación de un docente y suscribe la posibilidad de ofrecer un servicio educativo de mejor o menor calidad, dependiendo de la combinación que resulte de dichas condiciones.

\section{Referencias bibliográficas}

AHUMADA, P. Evaluación de la eficiencia docente. En: ALVAREZ MARTíN, F. Evaluación de la acción docente en Latinoamérica. Santiago de Chile: PREAL REDUC. 1997. Disponible en: www.reduc.cl/homereduc.nsf/08.245.

BAZÁN RAMÍREZ, A. Tomando en cuenta algunas variables que intervienen en el proceso de adquisición formal de la lengua escrita. En: RAMÍREZ, B. (Ed.) Enseñanza y evaluación de la lectura y escritura: algunos aportes de la investigación en psicología. México: Instituto Tecnológico de Sonora - Consejo Nacional de Ciencia y Tecnología. 2001. p. 81-96.

CAMBEROS, M.; GENESTA, M. A.; HUESCA, L. La pobreza en Sonora: Ios límites a la modernización. Revista de Estudios Sociales. v. 5, n. 9, p. 168-197. 1994.

CAZARES, A. (1995). La personalidad efectiva para la docencia efectiva. Pedagogía, v. 10, n. 3. Disponible en: www.oei.es/ buscador.htm.

De LA TORRE, S. Aprender de los errores: el tratamiento didáctico de los errores como estrategia de innovación. Madrid: Editorial Escuela Española. 1993.

EZPELETA, J.; WEISS, E. Cambiar la Escuela Rural: evaluación cualitativa del Programa para Abatir el Rezago Educativo. (Ed. Rev.) México: Centro de Investigación y de Estudios Avanzados del Instituto Politécnico Nacional. Departamento de Investigaciones Educativas. 2000.

FERNÁNDEZ PÉREZ, M. Las tareas de la profesión de enseñar práctica de la racionalidad curricular. España: Editorial Siglo XXI.1994.

GAJARDO, M.; De ANDRACA, A. M. Docentes y docencia: las zonas rurales. Informe de investigación realizada en las zonas rurales de Chile. Santiago, Chile: OREALC. 1992. Disponible en: www.reduc.cl/homereduc.nst/06.337-00).

GALO DE LARA, C. M. El maestro como orientador del aprendizaje: evaluación del desempeño. En: ALVAREZ MARTín, F. Evaluación de la acción docente en Latinoamérica. Santiago de Chile: PREAL. REDUC. 1997. Disponible en: www.reduc.cl/homereduc.nsf/ 08.245

GONZÁLEZ, D. et al. Relaciones entre variables de apoyo familiar, esfuerzo académico y rendimiento escolar en estudiantes de secundaria: un modelo estructural. Enseñanza e Investigación en Psicología, v. 3, n. 1, p. 163-181. 1998.

HAIR, J. et al. Análisis multivariante. Madrid: Prentice Hall. 2000.

IBARROLA, M.; SILVA, G.; CASTELÁN, A. ¿Quiénes son nuestros profesores?: en la investigación educativa en México, 19961997. En: CONGRESO NACIONAL DE INVESTIGACIÓN EDUCATIVA, 4., Resúmenes Analíticos... México: Consejo Mexicano de Investigación Educativa, A. C. 1997. p. 191-194. 
MCKINNEY, J. Tipología constructiva y teoría social. Trads. Hidegarde, B. y Torres, P. Buenos Aires: Editorial Amrrortu. 1968.

ROCKWELL, E. Desde la perspectiva del trabajo docente. México: CEE-REDUC. 1990. (Ponencia presentada en el Coloquio sobre el estado actual de la Educación en México)

SCHMELKES, S.; MUÑOZ IZQUIERDO, C. (1982). Los maestros de educación básica: estudios de mercado de trabajo. En: SCHMELKES. S. La calidad en la educación primaria: un estudio de caso. México: CFE. 1997.

SIERRA BRAVO, R. Técnicas de investigación social. España: Ed. Parainfo. 1985.

SPSS Guía del usuario del sistema de base de SPSS 7.5 para Windows. 1997. p. 97-203 y 301-306.

VELÁSQUEZ CASTAÑEDA, V. La evaluación como recurso para elevar la calidad de la educación en México. Revista lberoamericana de Educación, n. 10. 1996. Disponible en: www.oie.es.

VERA-NORIEGA, J. Á.; SERRANO, E. Efectos de desayunos escolares sobre diferentes dimensiones del lenguaje en niños preescolares de la zona rural de Sonora México. Revista Sonorense de Psicología, v. 12, n. 1, p. 1-13. 1998.

VERA- NORIEGA, J. Á. et al. Efectos de los desayunos escolares en la conducta de niños de primer grado de primaria. Enseñanza e Investigación en Psicología, v. 4, n. 1, p. 51-62. 1999.

ZARZAR CHARUR, C. La definición de objetivos de aprendizaje. Una habilidad básica para la docencia. Revista Perfiles Educativos, v. 63. 1994. Disponible en: www.unam.mx/cesu/perfiles/doc/63-02.pdf.

Recebido em 20.10.04

Aprovado em 03.03.05

Rosario Leticia Domínguez Guedea cursou licenciatura e mestrado na Universidade de Sonora. É docente universitária e tem participado de investigações orientadas para a educação básica em zonas urbanas marginais e zonas rurais. Atualment participa em projetos de investigação voltados para as temáticas da docência e avaliação institucional no ensino superior. e-mal: Idominguez@biblus.uson.mx.

José Ángel Vera Noriega cursou mestrado e doutorado em Psicologia Social na UNAM. É investigador do CIADAC, no México, membro do Sistema Nacional de Investigadores e do Conselgo Mexicano de Investigaçào Educativa. Atualmente elabora e avalia programas de educação básica numa perspectiva intercultural, etnopsicológica utilizando métodos qualitativos e quantitativos. 